\title{
High-Throughput Metabolomics Evaluate the Efficacy of Total Lignans From Acanthophanax Senticosus Stem Against Ovariectomized Osteoporosis Rat
}

\author{
Ai-hua Zhang ${ }^{1}$, Zhi-ming Ma ${ }^{1}$, Hui Sun ${ }^{1}$, Ying Zhang ${ }^{1}$, Jian-hua Liu ${ }^{1}$, Fang-fang $W u^{2}$ and \\ Xi-jun Wang ${ }^{1,2 *}$
}

${ }^{1}$ National TCM Key Laboratory of Serum Pharmacochemistry, Laboratory of Metabolomics, Department of Pharmaceutical Analysis, National Chinmedomics Research Center, Sino-America Chinmedomics Technology Collaboration Center, Heilongjiang University of Chinese Medicine, Harbin, China, ${ }^{2}$ National Engineering Laboratory for the Development of Southwestern Endangered Medicinal Materials, Guangxi Botanical Garden of Medicinal Plant, Nanning, China

\section{OPEN ACCESS}

Edited by:

Rong-Rong He,

Jinan University, China

Reviewed by:

Jian Li,

Beijing University of Chinese Medicine, China

Yong Tan,

Chinese Academy of Medical

Sciences, China

${ }^{*}$ Correspondence:

Xi-jun Wang

chinmedomics@126.com;

xijunwangls@126.com

Specialty section:

This article was submitted to

Ethnopharmacology,

a section of the journal

Frontiers in Pharmacology

Received: 22 December 2018

Accepted: 02 May 2019

Published: 29 May 2019

Citation:

Zhang A, Ma Z, Sun H, Zhang Y, Liu J,

Wu F and Wang $X$ (2019)

High-Throughput Metabolomics

Evaluate the Efficacy of Total Lignans

From Acanthophanax Senticosus

Stem Against Ovariectomized

Osteoporosis Rat.

Front. Pharmacol. 10:553

doi: 10.3389/fphar.2019.00553
Postmenopausal osteoporosis (PMOP) is a common clinical illness in postmenopausal women, but there is no effective drug at present. Metabolomics approach was used to explore the potential biomarkers of PMOP and evaluate the efficacy and therapeutic targets of total lignans in the stem of Acanthophanax senticosus (ASSL) on the ovariectomized osteoporosis model rats. UPLC/MS and pattern recognition methods were used for serum metabolites discovery to illustrate the pathological mechanism of PMOP model rats, and then revealing the intervention effect of ASSL. The pattern recognition result showed that serum metabolic profiles of the sham operation group and the model group were clustered clearly, and 16 potential biomarkers were finally identified (7 in positive ion mode and 9 in negative ion mode), and they are involved in 15 related metabolic pathways. After oral administration of ASSL, 10 biomarkers were found to be significantly up-regulated and mainly regulated metabolic pathways include unsaturated fatty acid biosynthesis, linoleic acid metabolism, and arachidonic acid metabolism, primary bile acid synthesis, tyrosine metabolism, etc. Our study demonstrated that the ASSL could affect the endogenous metabolites related metabolic mechanism, provides a pharmacological basis of the ASSL for PMOP treatment.

Keywords: metabolomics, biomarker, metabolic pathway, lignans, herbal medicine, efficacy, acanthophanax senticosus, ovariectomized osteoporosis

\section{INTRODUCTION}

Osteoporosis (OP) is a systemic bone disease characterized by bone mass reduction, bone strength reduction, and bone microstructure degeneration with the aging and aging of the human body, and it is also the main cause of high fracture incidence in recent years, which can lead to bone fragility and increased fracture risk (Wang et al., 2018; Watts, 2018). It has irreversible characteristics, and its early intervention methods have become a problem and focus of the whole society. It is divided into primary osteoporosis, secondary osteoporosis and idiopathic osteoporosis. Primary osteoporosis is divided into two categories, I -type primary osteoporosis is PMOP and type II osteoporosis is elderly osteoporosis; the former often occurs at 50 years old women in the left 
and right. After menopause, women's ovarian function declines, bone mass decreases, the rate of bone turnover in the body increases, and the rate of bone absorption exceeds the rate of bone formation, resulting in bone mass loss, bone density decrease and bone fragility increase, which is called PMOP (Horne et al., 2018). The animal model of postmenopausal osteoporosis (OP) can be established by classical castration surgery (Tella and Gallagher, 2014; Drake et al., 2015). At present, the combination of drugs and calcium therapy has a significant effect on senile osteoporosis, and estrogen combined with calcium therapy is better. Estrogen replacement therapy can increase estrogen levels in postmenopausal women, increase systemic bone mass and inhibit osteoclast activity, and slow bone metabolism, which can significantly reduce the incidence of fractures (Hassan et al., 2010). In recent years, some new drugs have also been used for the prevention and treatment of OP. However, early prevention and treatment are still difficult.

More and more Chinese herbal medicines have shown effective therapeutic effects on OP. Acanthopanax senticosus (AS) is derived from the rhizomes and stem of the Acanthopanax senticosus Rupr. Et Maxim. Harms, which was first published in $<$ Shen Nong Ben Cao $>$ (Huang et al., 2011). It has the functions of nourishing and strengthening, and contains a large number of chemical components, such as saponins, polysaccharides, lignins, flavonoids, organic acids, etc. (Hassan et al., 2010; Zhang et al., 2010b, 2016; Liu et al., 2012; Sun et al., 2014, 2016; Wang et al., 2017). In clinic, it play an excellent clinical effect in the cardiovascular system (Fujikawa et al., 1996, 2005). In addition, modern pharmacological studies have shown that AS also has anti-platelet aggregation, anti-thrombosis, anti-aging, antifatigue anti-inflammatory, and even anti-tumor effects (Zhang et al., 2010a). The AS stem is also medicinal part and has an estrogen-like anti-osteoporosis effect (Lee et al., 2004).

Metabolomics could explore the changes of all metabolites in living organisms after pathophysiological stimulation (Fiehn, 2002; Boudah et al., 2013). All metabolites were systematically analyzed by the high sensitivity and selectivity detection techniques (Sun et al., 2012; Wang et al., 2013b; Zhang et al., 2014a, 2015a; Yan et al., 2017), and metabolomics can qualitatively and quantitatively analyze endogenous small molecule metabolites in living organisms (Liu et al., 2017). UPLC/MS has been widely used in metabolite research practice because of its high sensitivity and high throughput characteristics (Dettmer et al., 2010; Zhang et al., 2011; Wang et al., 2015b). Multivariate analysis methods such as data processing and analysis techniques such as principal component analysis (PCA), partial least squares discriminant analysis (PLS-DA) and orthogonal partial least squares discriminant analysis (OPLS-DA) are widely used in metabolomics research (Song et al., 2017). In this study, the total lignans in the stem of Acanthopanax senticosus (ASSL) were used as the research object, and UPLC/MS technique was used to analyze all the metabolites and then illustrate the pathological mechanism, serum metabolomics method was used to explore the effect and identify potential therapeutic targets, provided a pharmacological basis of the ASSL for PMOP treatment.

\section{EXPERIMENTAL METHODS}

\section{Chemicals and Materials}

Alkaline phosphatase kit and tartrate-resistant acid phosphatase kit were purchased from the Institute of Bioengineering (Nanjing, China); Osteocalcin kit was purchased from Northern Biotechnology Research Institute (Beijing, China); sodium chloride injection was acquired from Sanlian Pharmaceutical Co., Ltd. (Harbin, China); iodophor disinfectant was purchased from Xinruida Disinfectant Co., Ltd. (De zhou, China); chloral hydrate and sodium carboxymethyl cellulose were obtained from the Institute of Photosynthetic Fine Chemicals (Tianjin, China); penicillin sodium powder injection from Harbin Pharmaceutical Group Pharmaceutical Factory; acetonitrile, methanol, acetone were obtained from Merck (chromatographic grade, Merck, Germany); Neil Estrone was purchased from Vikchi Biotech Co., Ltd. (Sichuan, China); other reagents and chemicals used were of analytical grade. Identification and structural characterization of compounds in the ASS was shown in Table S1 and Figure S1. We also had determined the content of total lignans in the ASS (Orchard et al., 2013).

\section{Animal Handling Procedures and Drug Treatment Model Preparation}

Experimental Animals: Clean SD female rats weighing $260 \pm$ $20 \mathrm{~g}$ were provided by the Experimental Animal Center of Heilongjiang University of Traditional Chinese Medicine. Pretest animals were conditioned for one week in a well-ventilated, quiet environment, with free access to water and standard feed. One week later, rats were randomly divided into the model group (OVX), sham operation group (SHAM), Nysterious group (NYL, $1 \mathrm{mg} / \mathrm{kg}$ ), and total lignans low dose group (ASSLL, $100 \mathrm{mg} / \mathrm{kg}$ ), total lignans medium dose group (ASSLM, $200 \mathrm{mg} / \mathrm{kg}$ ), total lignans high dose group (ASSLH, $400 \mathrm{mg} / \mathrm{kg}$ ) with 8 rats per group. Except for the sham operation group, the remaining 50 rats were fasted for $12 \mathrm{~h}$ before surgery and anesthetized with $10 \%$ chloral hydrate $(0.3 \mathrm{~mL} / 100 \mathrm{~g})$. The abdominal surface was fixed on the operating table, and the hair clipped range under the xiphoid process was $4 \times 3 \mathrm{~cm}$, and the surgical department was disinfected with iodine volts and $75 \%$ alcohol, respectively. The skin under the xiphoid process was $1.5 \mathrm{~cm}$, and a $2 \mathrm{~cm}$ long, $2-2.5 \mathrm{~cm}$ deep incision is cut longitudinally along the white line of the abdomen to separate the abdominal muscles from peritoneum and the abdominal cavity was exposed. White adipose tissue is clearly visible at the incision. The fat layer is opened and the uterus is found. Gently pull out one side of the uterine horn, and see the pink morular-shaped ovary wrapped by fat at the end. Clamp the fallopian tube below the ovary with tissue forceps, and ligate the fallopian tube and its surrounding vascular adipose tissue with the sheep intestine. The ovaries are removed, and the uterine horn is sent back to the abdominal cavity, and the other side of the ovaries is removed by the same method. The muscle layer was first sutured with the gut, and the skin layer was sutured with a medical wire. Once again, the skin suture was disinfected with iodophor, and 50,000 units of penicillin sodium were injected into the abdominal cavity. 
The sham operation group took the same amount of adipose tissue around the ovary and did not perform oophorectomy. The rats in each group were given intraperitoneal injection of sodium cyclamate for 7 consecutive days at the rate of 50,000 units per day. After recovery of surgical wounds in each group, gavage was started on the 7 th day. The sham operation group and the model group were given distilled water by gavage; the NYL group was intragastrically administered with nylestriol once a week, and distilled water was given by other time. The drug was administered at 8-10 a.m., and was administered continuously for 12 weeks. The dose was adjusted once a week according to the body weight.

\section{Sample Collection and Determination}

Rats in each group were fasted for $24 \mathrm{~h}$ after the last administration of the model for 12 weeks, and were free to drink water. After intraperitoneal injection of $10 \%$ chloral hydrate, $5 \mathrm{~mL}$ of blood samples were taken from the abdominal aorta, allowed to silence for $30 \mathrm{~min}$, centrifuged at 3,000 rpm for $10 \mathrm{~min}$ at $4{ }^{\circ} \mathrm{C}$, and the supernatant fluid was removed and dispensed into a $1.5 \mathrm{~mL}$ centrifuge tube and frozen at $-80^{\circ} \mathrm{C}$. To be used for the determination of biochemical indicators of bone metabolism and blood metabolic profiles. Cut the back skin of the rat, separate the lumbar vertebrae (LV1-6) from the rat body, remove the muscle attached to the vertebrae, cut off the intervertebral disc and tendon, and remove the lumbar vertebrae (LV1-4) and lumbar vertebrae (LV5), respectively. Gauze of saline was wrapped and frozen at $-20^{\circ} \mathrm{C}$ for the determination of the maximum load of bone density and bone biomechanical concave experiment. The uterus of each group was extracted, and the wet weight was weighed, according to the wet weight of the uterus (mg) /body weight (g) to calculate the uterus index.

\section{Metabolomics Profiling}

Serum samples were thawed at room temperature, $200 \mu \mathrm{L}$ serum samples were taken, vortexed for $10 \mathrm{~s}$, and add $800 \mu \mathrm{L}$ equal proportion solution of methanol, acetonitrile, acetone to precipitate the protein, vortexed for $30 \mathrm{~s}, 4^{\circ} \mathrm{C}, 13,000 \mathrm{rpm}$, centrifuged for $20 \mathrm{~min}$, and remove supernatant. Place in a $40^{\circ} \mathrm{C}$ water bath, blow dry with nitrogen, add $160 \mu \mathrm{L}$ of methanol to the residue, vortex for $60 \mathrm{~s}$, then add $40 \mu \mathrm{L}$ of water, vortex for $60 \mathrm{~s}$, centrifuge at $13,000 \mathrm{rpm}$ for $10 \mathrm{~min}$ at $4^{\circ} \mathrm{C}$, and take the supernatant through a $0.22 \mu \mathrm{m}$ filter filtrate for UPLC analysis. Sample chromatographic conditions: ACQUITY UPLCTM HSS T3 $(100 \mathrm{~mm} \times 2.1 \mathrm{~mm}$ id, $1.8 \mu \mathrm{m})$; the mobile phase consisting of $\mathrm{A}$ (water and $0.1 \%$ formic acid) and $\mathrm{B}$ (acetonitrile and $0.1 \%$ formic acid) was analyzed at a column temperature of $40^{\circ} \mathrm{C}$, a flow rate of $0.4 \mathrm{~mL} / \mathrm{min}$ and a sample volume of $2 \mu \mathrm{L}$. The gradient was set as follows: $0-5 \mathrm{~min}$ : $99-65 \% \mathrm{~A}, 5-8 \mathrm{~min}$ : $65-$ 45\% A, 8-9.5 min: 45-30\% A, 9.5-13 min: 30-0\% A, maintaining $0 \%$ A for $2 \mathrm{~min}$ for equilibration of the column. Electrospray ionization source (ESI) was used for a full scan in positive and negative ion mode. The conditions were set as follows: ion spray voltage was set to $5.5 \mathrm{KV}$ in positive ion mode and $4.0 \mathrm{KV}$ in negative ion mode; ion source temperature was $600^{\circ} \mathrm{C}$; the deconvolution voltage (DP) is $100 \mathrm{~V}$ and the collision energy (CE) is set to $35 \mathrm{eV}$. Nitrogen is the atomizing gas and the auxiliary gas, both are 55 psi and 65 psi in positive and negative ion modes, respectively, the blast gas is 35 psi.

\section{Multivariate Statistical Analysis and Data Processing}

UPLC-MS technology was used to analyze the pre-treated serum samples in the positive and negative ion mode according to the established conditions. Serum ESI-MS metabolic profile data was input into the Progenesis QI software for data preprocessing. After the peaks were matched, extracted and normalized, the ions were normalized and unsupervised PCA was performed on all ions. Then, supervised OPLS-DA is performed to calculate the contribution value of each ion reflecting the clustering and intergroup separation in the group, and $T$-testing the normalized data. Statistically significant ions with $P<0.05$ difference between groups were selected as candidate ions and element matching and secondary identification were performed.

\section{Biomarkers Identification and Metabolic Pathway}

Firstly, the Rt-m/z of the important metabolite ions is locked, and the ions are extracted in the TIC total ion chromatogram to extract the Mass, and then the elemental composition analysis is performed to determine the possible chemical formula. After that searched on the Human Metabolome Database (HMDB) and Metaboanalyst website, and initially matched the mass spectrometry information to estimate the possible attribution of the metabolites. Based on the massfragment software attached to the Masslynx software system, the obtained compounds and the secondary mass spectrum were used as prerequisites. The effectiveness of the metabolites was demonstrated by pyrolysis of the mass spectrometry and the possibility of chemical structure cleavage. MetPA (Metabolomics Pathway Analysis) is a specialized network tool for analyzing metabolic pathways with biological background in visual metabolomics data. MetPA combines a variety of advanced path analysis programs to analyze the topological characteristics of metabolite metabolic pathways to help identify a metabolic pathway most relevant to metabolomics research. Using this software, determine the serum metabolic pathway associated with the resulting biomarker.

\section{Statistical Analysis}

The data were treated with SPSS software, and the statistical results were represented by $(x \pm s)$. Univariate anova was used for inter-group comparison, and Bonferroni test was used for multiple comparison, $P<0.05$ was considered to be statistically significant. $P<0.01$ was considered to have an extremely significant statistical difference.

\section{RESULTS}

\section{Bone Mineral Density and Bone Biomechanics}

Place the lumbar vertebrae (LV1-4) under the probe of the bone densitometer (DPX-MD, America), scan it with small animal software (Figure 1A), and place the lumbar vertebrae (LV5) at the universal material testing machine (Zwick/Roell, Germany) the maximum load of the concave experiment was measured (Figure 1B). The results displayed that the index 


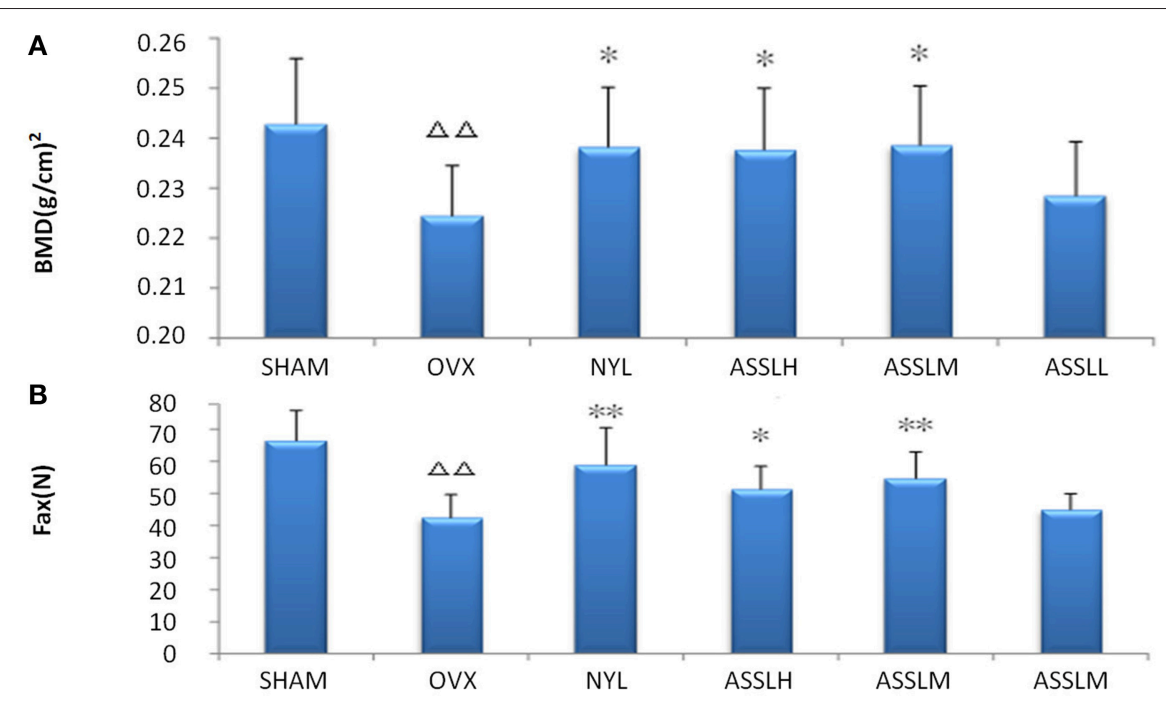

FIGURE 1 | Effects of nylestriol and total lignans on the bone mineral density and the maximum load of ovariectomized osteoporosis rat model (compared with sham group, ${ }^{\Delta} \Delta P<0.01$; the OVX model group, ${ }^{\star} P<0.05,{ }^{\star \star} P<0.01$ ) (A) bone mineral density (B) maximum load.

of the OVX group decreased compared with the SHAM group $(P<0.01)$. Compared with the OVX group, NYL and ASSLH, ASSLM group were increased, which was statistically significant $(P<0.01, P<0.05)$. The increase in the ASSLL group was lowly and it had no significant differences. Compared with the NYL, ASSLH, and ASSLM groups, the increase in bone density was similar, indicating that all three groups can improve bone mineral density reduction caused by ovariectomy, improve bone load capacity, and increase bone biomechanical properties.

\section{Bone Metabolism and Uterus Index Determination}

Serum alkaline phosphatase (AKP), serum tartrate-resistant acid phosphatase (TRAP) and serum osteocalcin (BGP) in bone metabolism were selected to evaluate the effect of ASSL on bone metabolism in ovariectomized rats. The results showed that compared with the SHAM, the AKP (Figure 2A), TRAP (Figure 2B), and BGP (Figure 2C) model groups were all increased $(P<0.01)$; compared with the OVX, NYL, ASSLH, ASSLM group can reduce the content of AKP, TRAP, BGP $(P<0.01, P<0.05)$, the ASSLL group has a small reduction, no statistical significance; NYL, ASSLH, ASSLM group can significantly reduce ovariectomy. The increase of bone metabolism index caused by surgery indicates that ASSL can effectively inhibit bone over-transformation, regulate bone cell balance, and inhibit osteoblast cell disruption. Therefore, it can increase bone formation and decrease bone resorption, thus reducing bone turnover and regeneration rate.

The uterus index was measured by extracting the uterus of the rat. The results showed (Figure 2D): compared with the SHAM group, the uterus index of the OVX group decreased $(P<0.01)$; compared with the OVX group, the uterus index of the NYL, ASSLH, and ASSLM groups increased. The ASSLL group had a small increase and was not statistically significant. An increase in the uterus index indicates an increase in estrogen levels, but the three did not restore the uterus index to its original level.

\section{Metabolic Profiling}

Using the above UPLC-MS conditions, the serum samples were processed in accordance with the serum sample processing method, and the serum samples were subjected to full-scan analysis in positive and negative ion mode. The serum ESI-MS metabolic profile data were input into the Progenesis QI software. After the peaks were matched, extracted and normalized, the ions were normalized, and all ions were analyzed in PCA to obtain the change between the groups. The trend of the score plot, positive (Figure 3A) negative (Figure 4A), showed that the osteoporosis model group and the sham operation group clustered significantly; indicating that the endogenous serum metabolic network of rats after membrane formation changed significantly. To find the endogenous metabolites that play a considerable role in the metabolism clustering separation, the above-mentioned serum sample metabolic profile was analyzed by OPLS-DA to obtain Score plot of positive mode (Figure 3B) and negative mode (Figure $4 \mathbf{B}$ ). The osteoporosis rat model group was significantly separated from the blank control group, and the S-plot diagram was further positive (Figure 3C), negative mode (Figure 4C), the farther away from the far point in the figure, the greater the contribution rate. Further select ions with VIP value $>1$ in the VIP scatter plot as showed in the positive mode (Figure 3D) and negative mode (Figure 4D), and then combined with the VIP value $>1$ and the inter-group $T$-test results, ions with a $P$-value of $<0.05$ were selected for structural identification as potential biomarkers. The metabolic profile fingerprint of the serum sample is shown in Figures 5, 6.

\section{Metabolite Identification}

Lock the Rt_m/z of the important metabolite ions, and obtain the ions in the TIC total ion chromatogram to extract the 

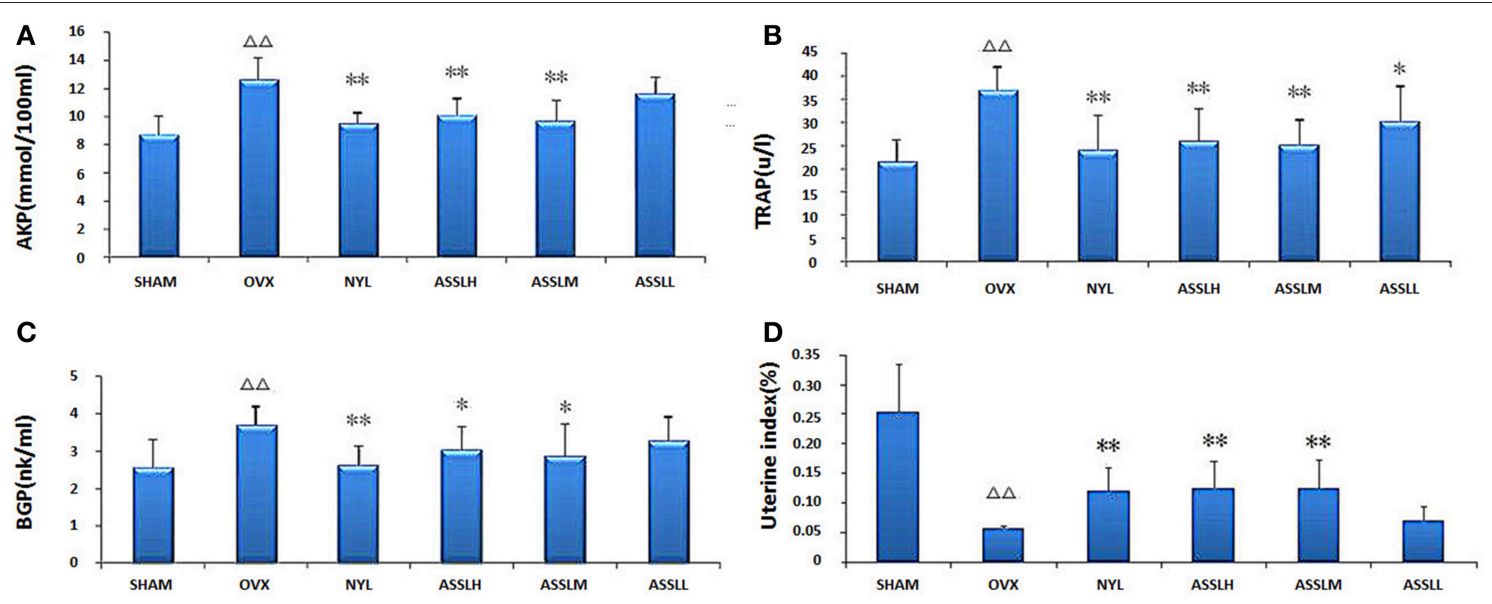

FIGURE 2 | Effects of nylestriol and total lignans on the serum alkaline phosphatase, tartrate-resistant acid phosphatase and osteocalcin of ovariectomized osteoporosis rat model (compared with sham operation group, ${ }^{\Delta \Delta} P<0.01$; the OVX model group, ${ }^{\star} P<0.05$, ${ }^{\star *} P<0.01$ ). (A) Alkaline phosphatase; (B) Tartrate-resistant acid phosphatase; (C) Osteocalcin; (D) Rat uterus index.

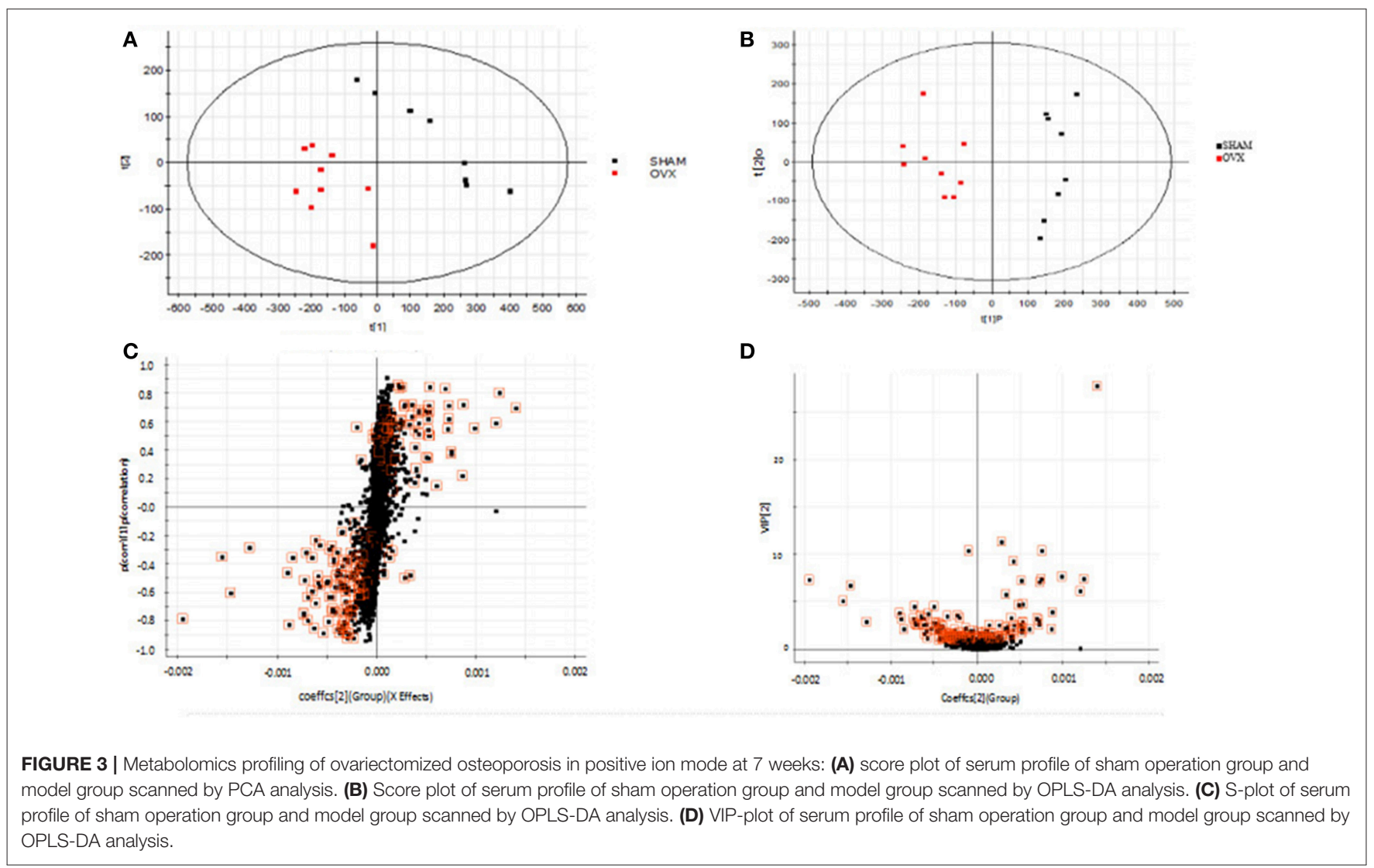

Mass, and perform element composition analysis to determine the possible chemical formula. Based on the possible chemical formulas, accurate masses and secondary mass spectrometry data (MS/MS) of ions, the Human Metabolome Database (HMDB) and Metaboanalyst sites were searched to match the mass spectrometry information, and the possible attribution of metabolites was speculated. The effectiveness of the metabolites was demonstrated using the massfragment software included with the masslynx software system. In this study preliminarily identified 16 key metabolites, as showed in Table S2. The results of the secondary ion fragmentation information matching of these 16 compounds are shown in Table S3. The content of these 
A
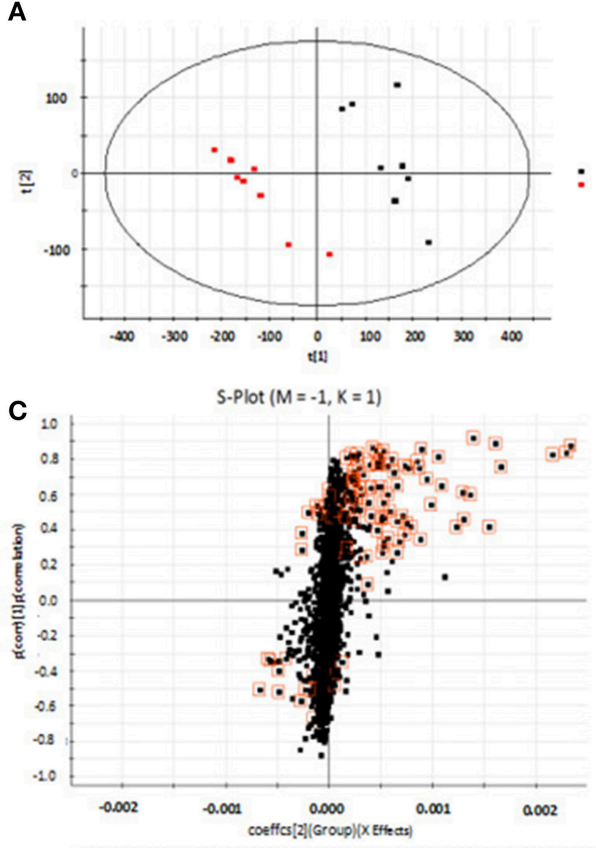

B

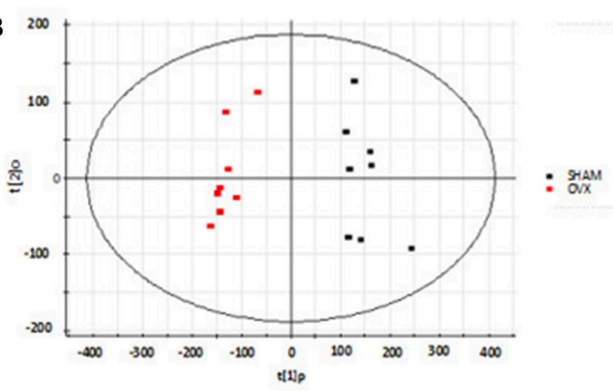

D

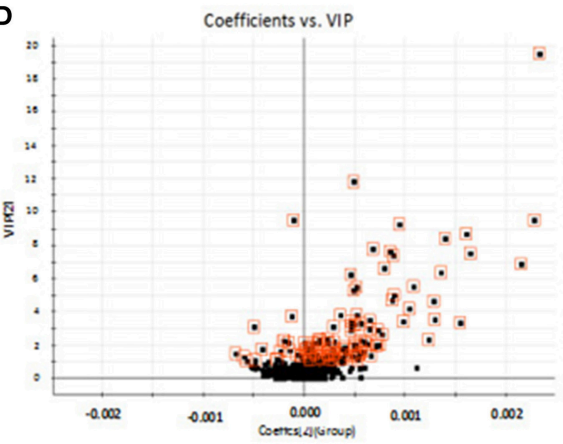

FIGURE 4 | Metabolomics profiling of ovariectomized osteoporosis in negative ion mode at 7 weeks: (A) score plot of serum profile of sham operation group and model group scanned by PCA analysis. (B) Score plot of serum profile of sham operation group and model group scanned by OPLS-DA analysis. (C) S-plot of serum profile of sham operation group and model group scanned by OPLS-DA analysis. (D) VIP-plot of serum profile of sham operation group and model group scanned by OPLS-DA analysis.
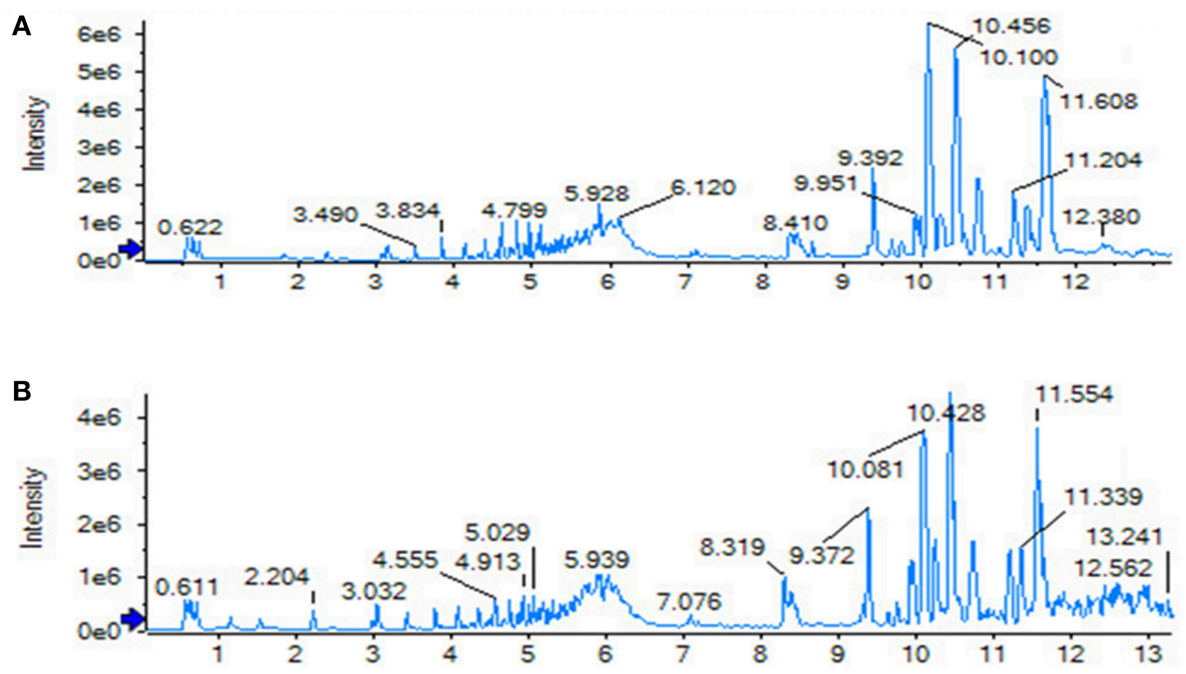

FIGURE 5 | Serum TIC map in positive ion mode. (A) Sham operation group; (B) model group.

potential biomarkers in the 12th week model group and the 12th week blank group were statistically analyzed (Figure 7).

\section{Biological Pathway and Functional Analysis}

MetPA (Metabolomics Pathway Analysis) is a specialized network tool for analyzing metabolic pathways with biological background in visual metabolomics data. MetPA combines many advanced path analysis programs to analyze the topological characteristics of metabolic pathways of metabolites can helps to determine one metabolic pathway most relevant to metabolomics research. MetPA analysis was performed on the identified 16 biopsy markers related to osteoporosis model, and 15 related metabolic pathways were obtained, 


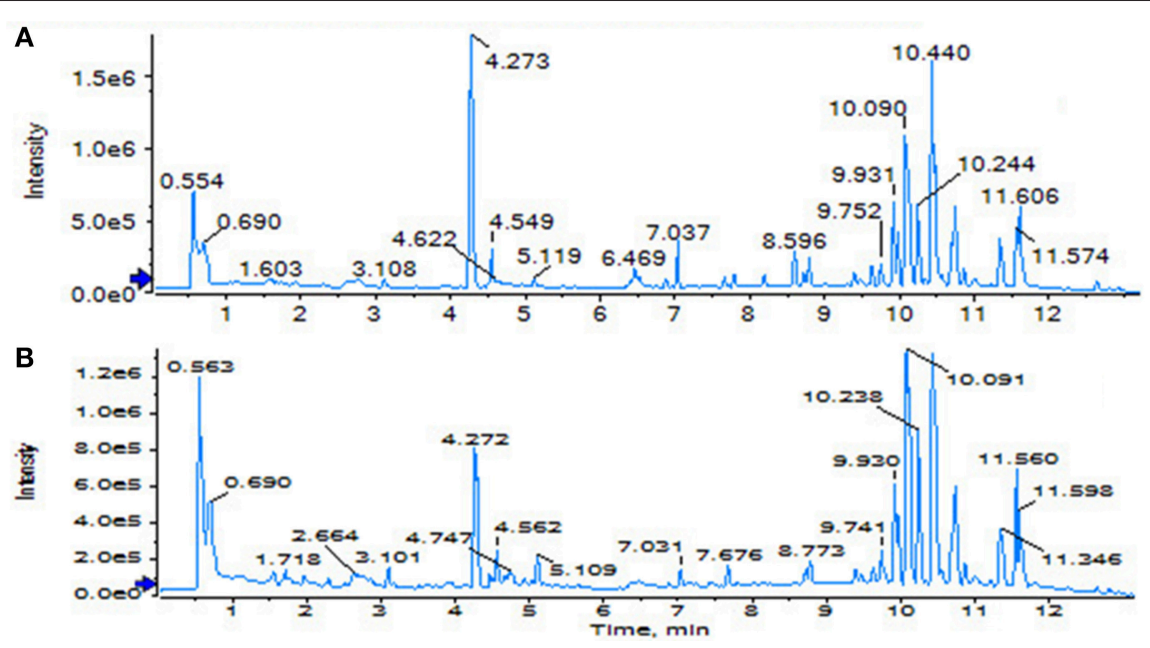

FIGURE 6 | Serum TIC map in negative ion mode. (A) Sham operation group; (B) model group.

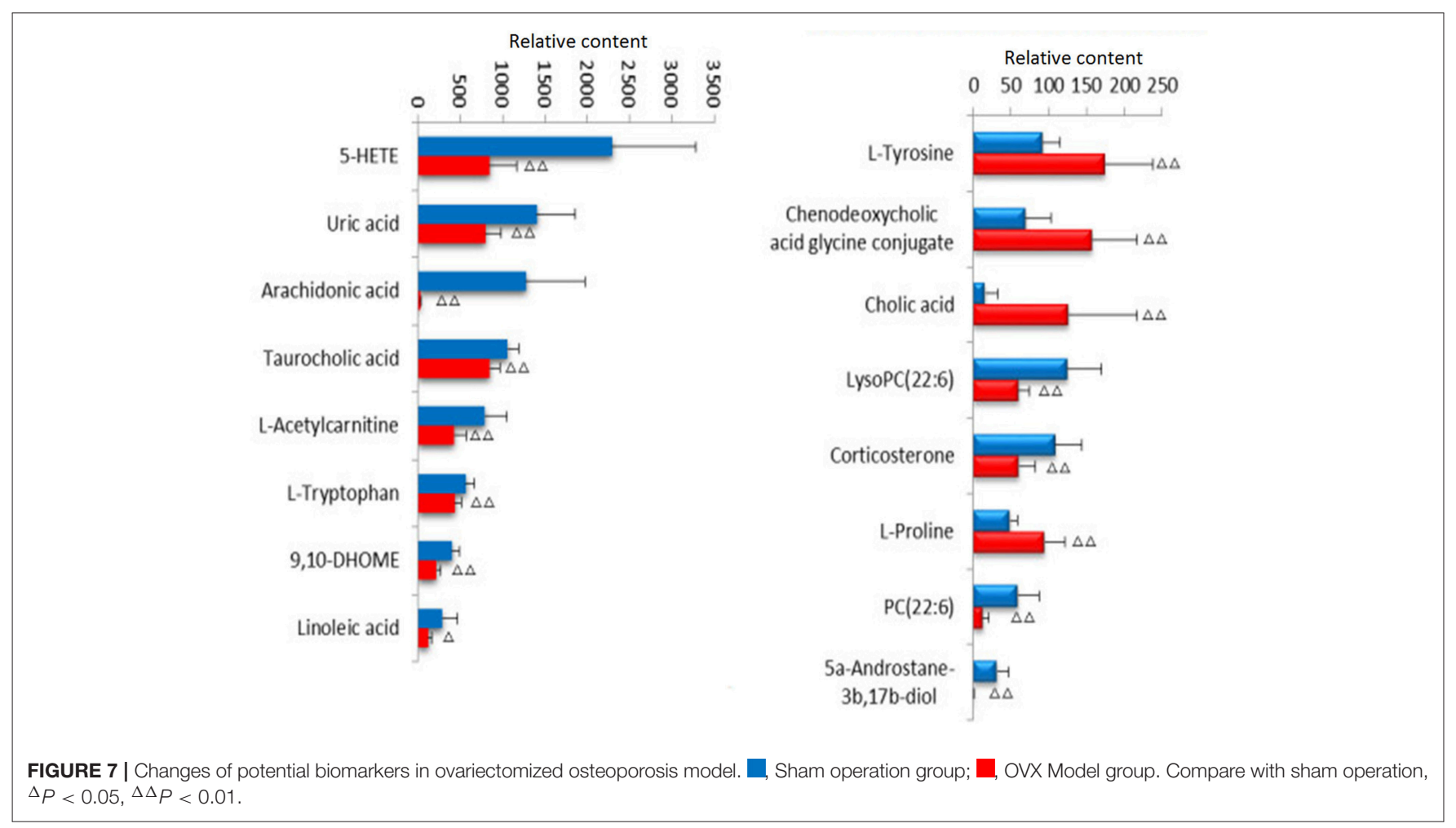

including Aminoacyl-tRNA biosynthesis; ubiquinone and other terpenoid-quinone biosynthesis; phenylalanine, tyrosine and tryptophan biosynthesis; primary bile acid biosynthesis; taurine and hypotaurine taurine and hypotaurine metabolism; Arachidonic acid metabolism; glycerol phospholipid metabolism; biosynthesis of unsaturated fatty acids; tyrosine metabolism; fine Arginine and proline metabolism; purine metabolism; linoleic acid metabolism; tryptophan metabolism; steroid hormone biosynthesis, alpha-linolenic acid metabolism see Figure 8 for details. These results suggest that these endogenous metabolites strongly perturb the whole metabolic trajectory and are closely related to ovariectomized osteoporosis. Three closely related metabolic pathways were obtained, namely lipid metabolism, amino acid metabolism and nucleotide metabolism.

\section{Therapeutic Effects Analyses on Metabolite Profile}

The SHAM, ASSLH, ASSLM, ASSLL group of rats in the 12th week blood samples were processed according the foregoing method, positive and negative ions are completely scanned, 


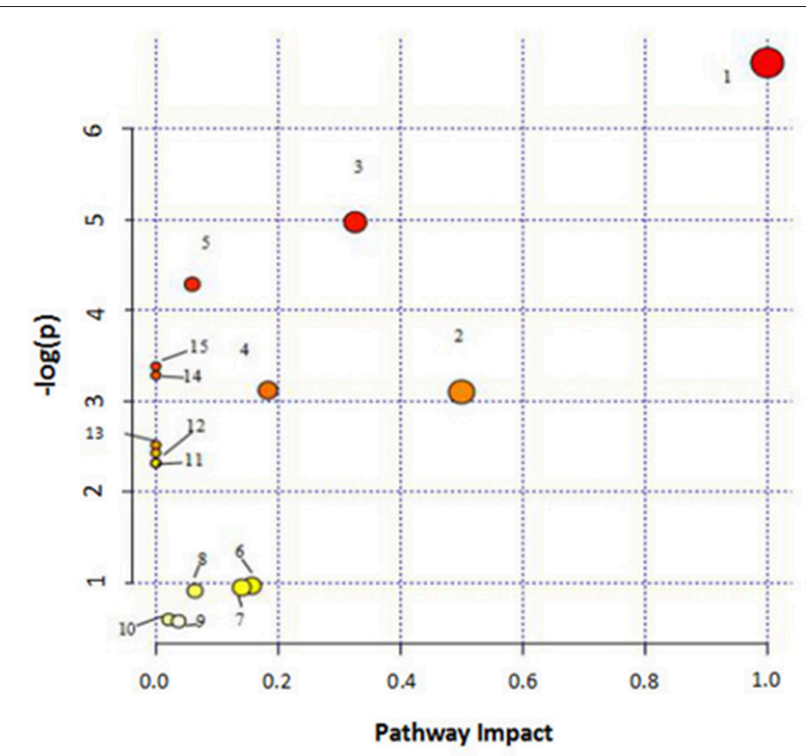

FIGURE 8 | Main metabolic pathways of potential biomarkers. Linoleic acid metabolism; (2) Phenylalanine, tyrosine and tryptophan biosynthesis; (3) Arachidonic acid metabolism; (4) Glycerophospholipid metabolism; (5) Primary bile acid biosynthesis; (6) Tryptophan metabolism; (7) Tyrosine metabolism; (8) Arginine and proline metabolism; (9) Steroid hormone biosynthesis; (01) Purine metabolism; (11) alpha-Linolenic acid metabolism; (12) Taurine and hypotaurine metabolism; (13) Biosynthesis of unsaturated fatty acids; (14) Aminoacyl-t RNA biosynthesis; (15) Ubiquinone and other terpenoid-quinone biosynthesis.

and the metabolic profile of the three-dimensional information (retention time, mass-to-nucleus ratio, peak intensity) of each group is obtained as shown in Figures 9, 10. The blood metabolic profile data of the 12th week of each test group were imported into the QI software for data dimensionality reduction and mass spectrometry matrix information acquisition. Further, the EZinfo2.0 software module was used to perform unsupervised Principal Components Analysis (PCA) analysis on each group of data. Figure 11 shows the score plot showing the trend of changes between groups. It can be seen that the clusters in each group were clustered and separated between groups; The sham operated group was significantly separated from the ovariectomized osteoporosis model, indicating that the rat serum metabolism profile was significantly different from that of the sham operated rats after replication of the ovariectomized osteoporosis rat model; ASSLH and ASSLM intervention group clustering, and the vector position is closer to the SHAM group, which indicated that ASSL could delay the pathological process of ovariectomized osteoporosis model rats to some extent.

\section{Effects on Potential Biomarkers}

To analyze the changes of the potential biomarkers of osteoporosis model induced by ovariectomy in different doses of ASSL enrichment and oral administration of positive control drugs, It was found that the application of ASSL enrichment intervention can affect the potential biomarkers of osteoporosis model induced by ovariectomy, and its content is adjusted back to the direction of the sham operation group. Among the 16 potential biomarkers identified, nylestriol intervention could be adjusted back to 11 and 7 were statistically different; the ASSLH enrichment could be adjusted back to 11 and 8 were statistically different; the dose intervention of ASSLM enrichment could be adjusted back to 11 and 7 were statistically different. The ASSLL enrichment could be adjusted back to 10 and 3 were statistically different. There are 10 common markers that can be called back at three doses, respectively phospholipids (PC (22:6 (4Z, 7Z, 10Z, 13Z, 16Z, 19Z)/18:4 (6Z, 9Z, 12Z, 15Z))), L-proline, uric acid, L-tyrosine, taurocholic acid, chenodeoxycholic acid glycine conjugate, cholic acid, phospholipids (LysoPC (20:3 (5Z, 8Z, 11Z))), arachidonic acid (5-HETE), linoleic acid (Figure 12). Studies on the metabolic pathways of potential biomarkers showed that the main metabolic pathways were lipid metabolism, amino acid metabolism, and nucleotide metabolism. It is suggested that the ASSL exert its role in preventing and treating osteoporosis by interfering with the above metabolic pathways.

\section{DISCUSSION}

PMOP is a common disease associated with aging, mainly in postmenopausal women. Due to lack of estrogen, bone mass is reduced and bone tissue structure changes, bone fragility is increased, fracture is easy, and pain and bone deformation caused by fracture the occurrence of comorbidities and other serious effects on life of the elderly. Bone mineral density, bone biomechanics and bone metabolism indicators are still important indicators for evaluating postmenopausal osteoporosis. In this study, a classic postoperative model of osteoporosis was established by classical surgical castration. The study found that at 12 weeks after model replication, the bone density of LV1-4 in the model group decreased significantly compared with the SHAM group $(p<0.01)$, the maximum load of LV5 concave experiment was significantly decreased $(p$ $<0.01)$, AKP, RTAP, BGP in rat serum increased significantly $(p<0.01)$, and the uterus index decreased significantly $(p<$ 0.01 ). Changes in these indicators indicate that ovariectomy surgery replicates the postmenopausal osteoporosis model, which results in decreased bone density, decreased bone strength, increased bone resorption, increased metabolic bone formation, and uterine atrophy. In the rats treated with different doses ASSL, the bone mineral density and bone biomechanical properties of the rats in each administrative group increased, and the serum levels of AKP, RTAP, and BGP decreased, which significantly increased the uterus index. The role of the lignans group in ASSL was extremely significant.

Metabolomics analysis was used to analyze the serum of ovariectomized osteoporosis model rats and blank control group to study the difference of endogenous small molecule metabolic profiles. It can be seen that the 12th week after model replication. Model group and blank group Clustering grouping is obvious. By querying $\mathrm{HMDB}$, MetPA, KEGG, and other databases, 16 potential biomarkers with significant influence on clustering group are locked. Related metabolic pathways involve lipid metabolism, amino acid metabolism, nucleic acid metabolism, etc., the roadmap of biomarkers and metabolic pathways is shown 


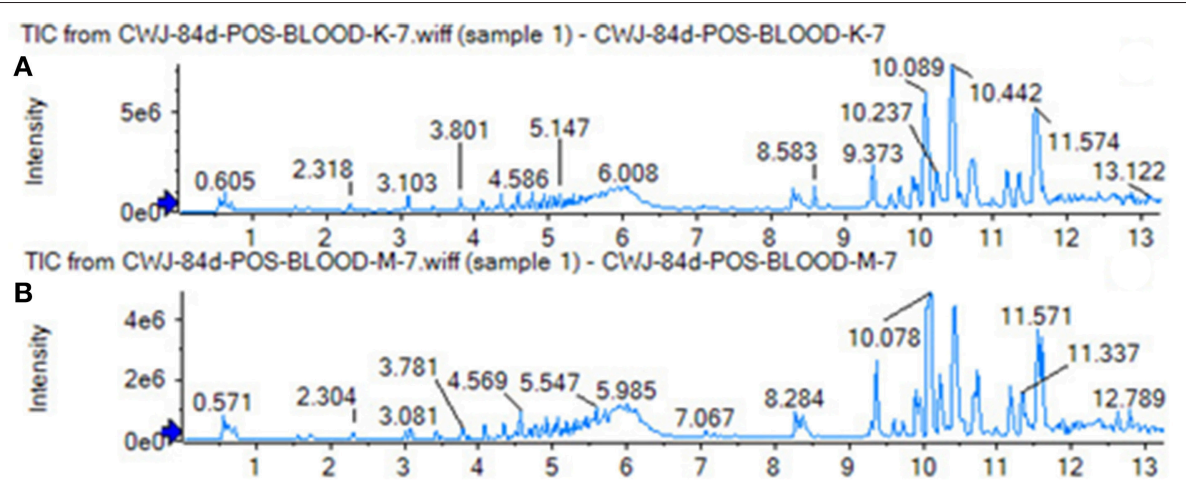

TIC from CWJ-84d-POS-BLOOD-Y-7. wiff (sample 1) - CWJ-84d-POS-BLOOD-Y-7

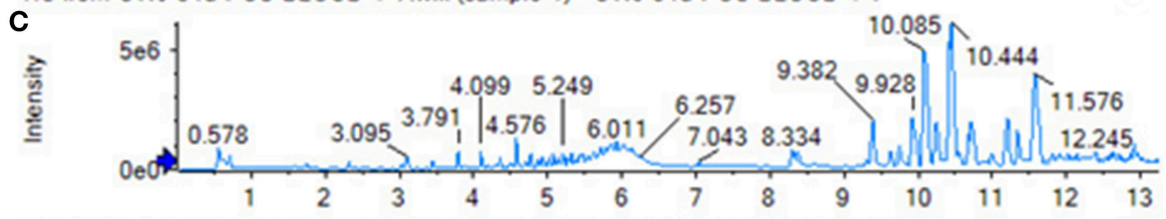

TIC from CWJ-84d-POS-BLOOD-H-5.wiff (sample 1) - CWJ-84d-POS-BLOOD-H-5

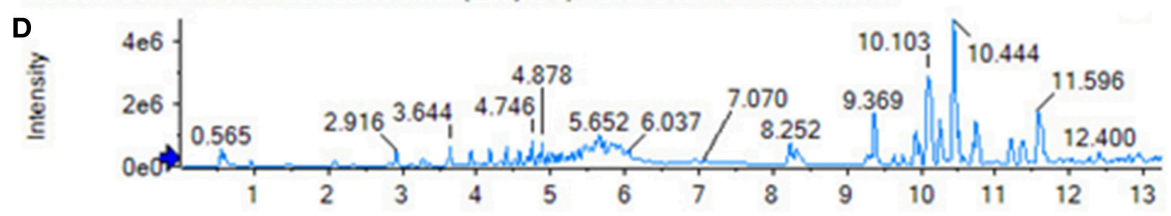

TIC from CWJ-84d-POS-BLOOD-CM-6. wiff (sample 1) - CWJ-84d-POS-BLOOD-CM-6

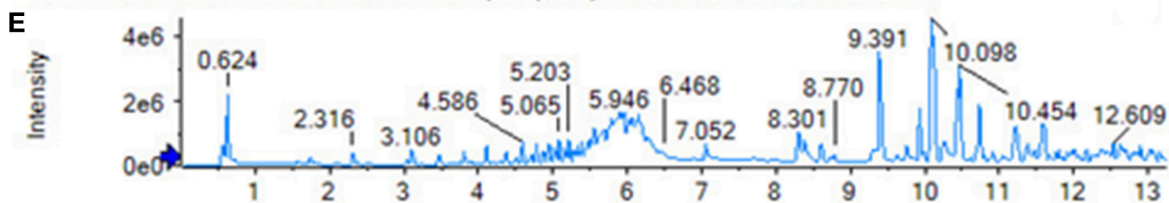

TIC from CWJJ-84d-POS-BLOOD-L-7.wiff (sample 1) - CWJ-84d-POS-BLOOD-L-7

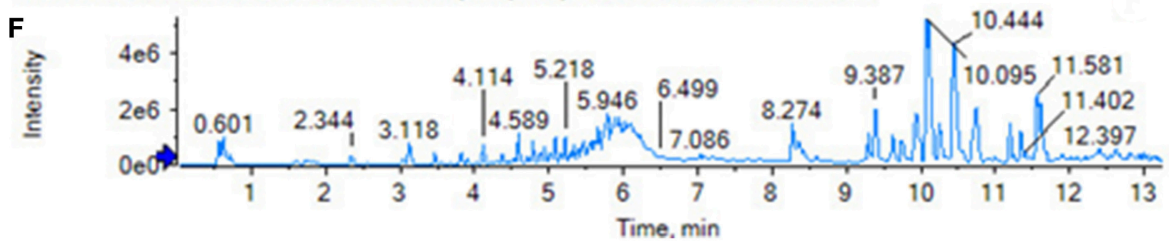

FIGURE 9 | TIC of total lignans on the ovariectomized osteoporosis rat model in positive ion mode. (A) Sham operation group; (B) OVX Model group; (C) NYL; (D) ASSLH; (E) ASSLM; (F) ASSLL.

(Figure 13), suggesting that the above metabolic pathway may be a potential biochemical mechanism of PMOP. After oral administration of different doses of ASSL, it was preliminaries determined that 10 biomarkers that can be adjusted in the high, medium and low dose groups of ASSL were associated with key metabolites by tracking. The metabolic pathway changes, and the main metabolic pathways involved ASSL include lipid metabolism, amino acid metabolism, and nucleotide metabolism.

Unsaturated fatty acids are composed of monounsaturated fatty acids and polyunsaturated fatty acids, which are essential fatty acids for human body. Polyunsaturated fatty acids include arachidonic acid, linolenic acid, and linoleic acid. Among them, linoleic acid can be converted into arachidonic acid in the body, which is called n6 series. Related studies have shown that n6 series polyunsaturated fatty acids can reduce the incidence of fractures (Orchard et al., 2013). Because it has the effect of promoting the growth of bone cells and increasing bone mineral density, unsaturated fatty acid metabolism disorder can cause osteoporosis (Harris et al., 2015). In this research, The researchers observed that the content of linoleic acid in the blood of rats was decreased after ovariectomy in rats, and it was speculated that the biosynthesis of unsaturated fatty acids was destroyed, which may lead to osteoporosis.

Amino acid metabolism is a relatively important part of life activities which is the basic unit of macromolecular protein. It is indispensable for normal metabolism in the human body. Some amino acids can also be transformed in the body. Related literature has shown that amino acids are beneficial 
TIC from CWJ-84d-NEG-BLOOD-K-7. wiff (sample 1) - CWJ-84d-NEG-BLOOD-K-7

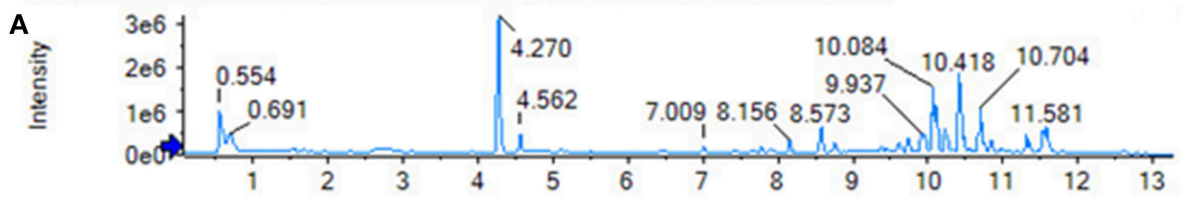

TIC from CWJ-84d-NEG-BLOOD-M-7.wiff (sample 1) - CWJ-84d-NEG-BLOOD-M-7

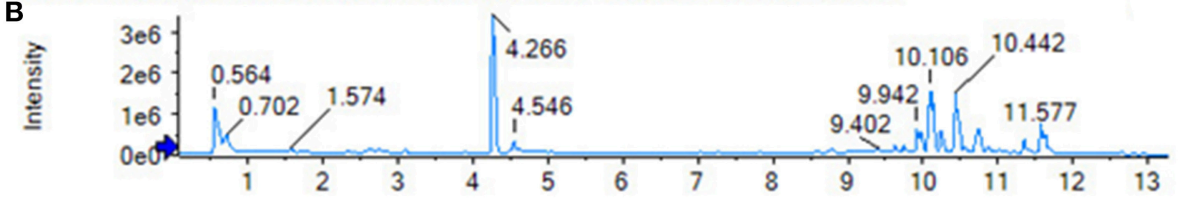

TIC from CWJ-84d-NEG-BLOOD-Y-7. wiff (sample 1) - CWJ-84d-NEG-BLOOD-Y-7

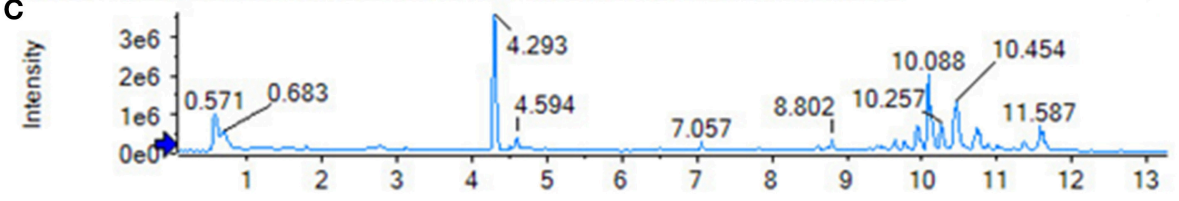

TIC from CWJ-84d-NEG-BLOOD-H-5.wiff (sample 1) - CWJ-84d-NEG-BLOOD-H-5

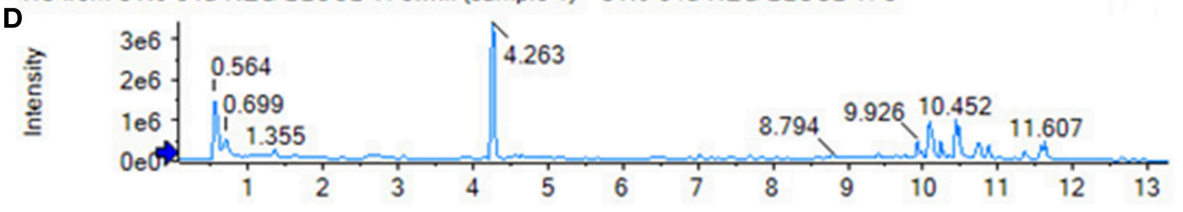

TIC from CWJ-84d-NEG-BLOOD-CM-6.wiff (sample 1) - CWJ-84d-NEG-BLOOD-CM-6

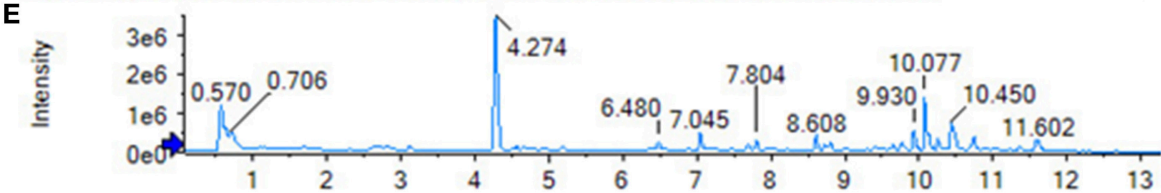

TIC from CWJ-84d-NEG-BLOOD-L-7.wiff (sample 1) - CWJ-84d-NEG-BLOOD-L-7

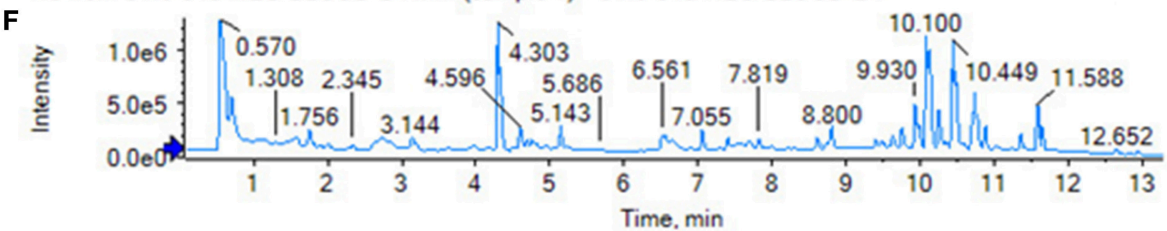

FIGURE 10 | TIC of total lignans on the ovariectomized osteoporosis rat model in negative ion mode. (A) Sham operation group; (B) OVX Model group; (C) NYL; (D) ASSLH; (E) ASSLM; (F) ASSLL.

for the treatment of osteoporosis (Torricelli et al., 2003). Amino acids are synthetic raw materials for hemoglobin and leukocytes, which have a certain effect on the prevention of osteoporosis in middle-aged and elderly people. When amino acid metabolism is abnormal, it will accumulate in the body, affecting the synthesis of proteins and hindering the synthesis of proteins in bone. Tyrosine is an important amino acid in the body and can produce a variety of metabolites. It is a major raw material for the synthesis of thyroxine. In the tyrosine metabolic pathway of this experiment, L-tyrosine content in ovariectomized osteoporosis model rats was higher than that in the blank group. It is speculated that the tyrosine content in the model group is increased, so in the model group thyroid gland the level of the hormone may also be elevated, indicating that it is abnormal in the synthesis, storage, and release in the body, and the hormone levels and biochemical indicators in the hypothalamic-pituitary-thyroid axis are disordered. Related literature has shown that excessive thyroxine can cause osteoporosis, which is consistent with related literature reports (Leb et al., 1994). Proline in bone organic matter is a key component of collagen. Proline (Szabados and Savouré, 2010) can increase the activity of acid phosphatase (ACP) in blood and increase the activity of alkaline phosphatase (ALP) of the osteoporosis model. This indicates that the estrogen level of the rat ovary is reduced and the bone turnover is accelerated. 

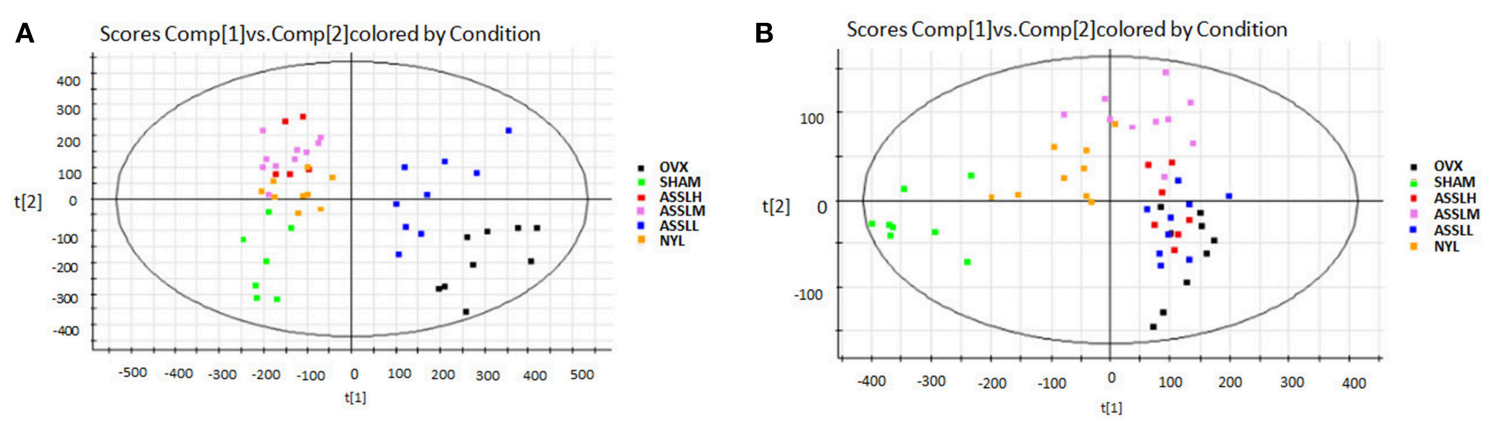

FIGURE 11 | PCA scores plot of metabolic profile of total lignans on the ovariectomized osteoporosis rat model in both positive and negative mode. (A) Positive ion mode; (B) Negative ion mode.
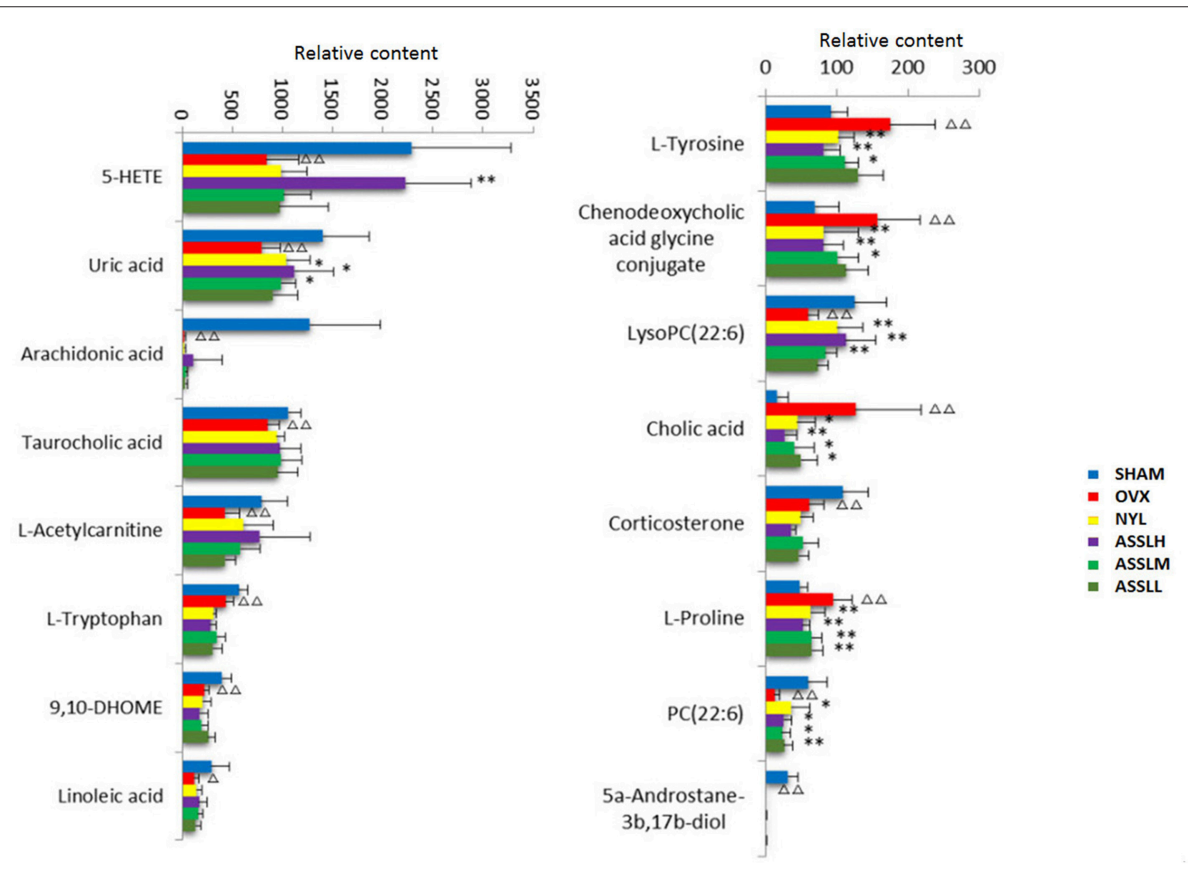

FIGURE 12 | Changes of potential biomarkers in total lignans on the ovariectomized osteoporosis rat model. $\square$, Sham operation group; $\square$, OVX Model group;, NYL; ASSLH; , ASSLM; aSSLL (Compare with sham group, ${ }^{\Delta} P<0.05, \Delta \Delta P<0.01$; compare with OVX Model group, ${ }^{\star} P<0.05$, ${ }^{\star *} P<0.01$ ).

Uric acid, as the final product of purine metabolism, is the main indicator of purine metabolism. Blood uric acid is a reducing substance in the body, which participates in the redox reaction in the body, has strong antioxidant capacity, and can scavenge free radicals against DNA damage (Johnson et al., 2003; Sautin and Johnson, 2008; Chen et al., 2015). Epidemiological studies have reported the possible effects of uric acid on osteoporosis. Blood uric acid may have a dual effect on bone. It is generally considered that physiological concentration of blood uric acid has an anti-osteoporosis effect. On the one hand, uric acid has a certain protective effect on bone metabolism, which is believed to be related to its antioxidant function (Ahn et al., 2013; Beyazit and Pek, 2018). Uric acid is also positively correlated with the level of parathyroid hormone (PTH) (Chikura et al., 2009; Hui et al., 2012) and can also affect bone metabolism by regulating the activity of $1 \alpha$-hydroxylase. On the other hand, oxidative stress reaction can lead to bone loss and participate in the mechanism of osteoporosis (Wauquier et al., 2009), which may be the crystallization deposition of uric acid, which reduces the activity of alpha-hydroxylase in the kidney and reduces the ability of the intestinal tract to absorb calcium.

Metabolomics is a newly developed discipline that mainly studies small molecular metabolites $(\mathrm{MW}<1000)$ as substrates and products of various metabolic pathways, it is a branch of system biology based on group index analysis, high-throughput detection and data processing, and aiming at information modeling and system integration (Wu et al., 2018). Metabolomics plays an important role in exploring metabolic disorder metabolism-related diseases (Zhang et al., 2013b, 2014b,c,d, 2018a; Wang et al., 2014; Liu et al., 2018; Sun et al., 2018), 


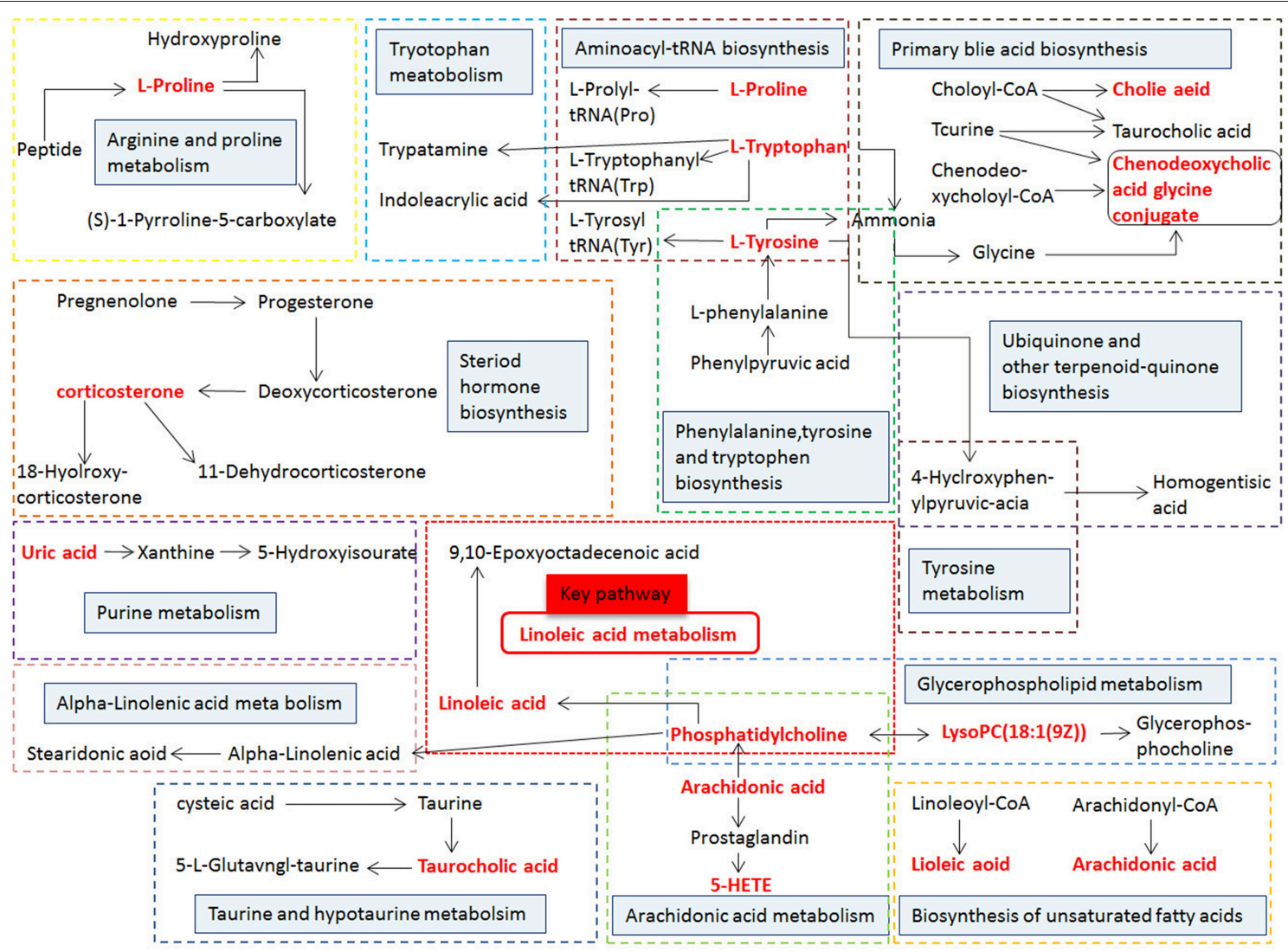

FIGURE 13 | Correlation networks of the potential biomarkers based on the KEGG. The red font represents the biomarkers detected in this experiment and blue filled boxes represent related metabolic pathways.

biomarkers identification (Wang et al., 2015a; Zhang et al., 2015b,c; Zhao et al., 2016; Song et al., 2017; Ren et al., 2018) and response to treatment (Wu et al., 2011; Dong et al., 2012; Sun et al., 2013; Wang et al., 2013a, 2016; Zhang et al., 2013a, 2018b). In this study, the metabolomics study was used to analyze serum metabolism of the ovariectomized bone rat model and to observe the changes of ASSL during the treatment. Sixteen potential differential metabolic markers were found to be associated with postmenopausal osteoporosis, refer to 15 metabolic pathways. After treatment with ASSL, 10 biomarkers can be significantly adjusted (Table S4) to regulate the biosynthesis of unsaturated fatty acids, linoleic acid metabolism, arachidonic acid metabolism, arachidonic acid metabolism, arachidonic acid metabolism, tyrosine metabolism, purine metabolism, glycerol phosphoric acid metabolism, etc., and the metabolic expression is in the direction of delaying PMOP. For the first time, the prevention and treatment of oral ASSL on PMOP were described at the level of metabolic pathways, and the effectiveness and time potential of metabolomics methods for the study of traditional Chinese medicine interventions was demonstrated.

\section{CONCLUSION}

In this study, the high-throughput metabolomics method was used to evaluate the efficacy of total lignans from
ASS against ovariectomized osteoporosis rat, and 16 potential biomarkers were finally identified and involved in 15 related metabolic pathways. ASSL could regulate 10 biomarkers of them and mainly adjusts metabolic pathways include unsaturated fatty acid biosynthesis, linoleic acid metabolism, and arachidonic acid metabolism, primary bile acid synthesis, tyrosine metabolism, etc. It showed that the ASSL could affect the endogenous metabolites related metabolic mechanism, offers a pharmacological basis of the ASSL for PMOP treatment, and provide evidences for the development of new drugs for PMOP.

\section{ETHICS STATEMENT}

The experimental procedures were approved by the Animal Care and Ethics Committee at Heilongjiang University of Chinese Medicine and all experiments were performed in accordance to the declaration of Helsinki.

\section{AUTHOR CONTRIBUTIONS}

$\mathrm{XW}$ conceived and designed the experiments. AZ, ZM, HS, $\mathrm{YZ}$, and JL performed the experiment. ZM, HS, YZ, and JL analyzed the data. $\mathrm{AZ}$ guided the experiment. $\mathrm{ZM}$ wrote the paper. AZ revised the paper. All authors read and approved the final manuscript. 


\section{FUNDING}

This work was supported by grants from the National Key Research and Development Program of China (2018YFC1706103), Key Program of Natural Science Foundation of State (Grant No. 81830110, 8181101160, 81430093, 81673586, $81703685,81302905,81503386,81373930)$, National Key Subject of Drug Innovation (Grant No. 2015ZX09101043-005, 2015ZX09101043-011), TCM State Administration Subject of Public Welfare (Grant No. 2015468004), Major Projects of Application Technology Research and Development Plan in Heilongjiang Province (GX16C003), Young Talent Lift

\section{REFERENCES}

Ahn, S. H., Lee, S. H., Kim, B. J., Lim, K. H., Bae, S. J., Kim, E. H., et al. (2013). Higher serum uric acid is associated with higher bone mass, lower bone turnover, and lower prevalence of vertebral fracture in healthy postmenopausal women. Osteopor. Int. 24, 2961-2970. doi: 10.1007/s00198-013-2377-7

Beyazit, F., and Pek, E. (2018). Effects of vitamin B12, folate, uric acid, and serum biomarkers of inflammation on bone mineral density in postmenopausal women. Prz Menopauzalny Menopause Review. 17, 69-76. doi: $10.5114 / \mathrm{pm} .2018 .77305$

Boudah, S., Paris, A., and Junot, C. (2013). Chapter four-liquid chromatography coupled to mass spectrometry-based metabolomics and the concept of biomarker. Adv. Bot. Res. 67, 159-218. doi: 10.1016/B978-0-12-397922-3.00004-6

Chen, L., Peng, Y., Fang, F., Chen, J., Pan, L., and You, L. (2015). Correlation of serum uric acid with bone mineral density and fragility fracture in patients with primary osteoporosis: a single-center retrospective study of 253 cases. Int. J. Clin. Exp. Med. 8, 6291-6294.

Chikura, B., Sadananda, V., and Usman-Saeed, M. (2009). Comment on: screening for Mycobacterium tuberculosis prior to anti-TNF therapy-an audit of impact of the British Thoracic Society guidelines on rheumatology practice in an area of low Mycobacterium tuberculosis prevalence. Rheumatology. 48, 1331-1332. doi: 10.1093/rheumatology/kep181

Dettmer, K., Aronov, P. A., and Hammock, B. D. (2010). Mass spectrometry-based metabolomics. Mass Spectrom. Rev. 26, 51-78. doi: 10.1002/mas.20108

Dong, W., Wang, P., Meng, X., Sun, H., Zhang, A., Wang, W., et al. (2012). Ultra-performance liquid chromatography-high-definition mass spectrometry analysis of constituents in the root of radix stemonae and those absorbed in blood after oral administration of the extract of the crude drug. Phytochem. Anal. 23, 657-667. doi: 10.1002/pca.2370

Drake, M. T., Clarke, B. L., and Lewiecki, E. M. (2015). The pathophysiology and treatment of osteoporosis. Clin. Ther. 37, 1837-1850. doi: 10.1016/j.clinthera.2015.06.006

Fiehn, O. (2002). Metabolomics-the link between genotypes and phenotypes. Plant Mol. Biol. 48, 155-171. doi: 10.1023/A:1013713905833

Fujikawa, T., Miguchi, S., Kanada, N., Nakai, N., Ogata, M., Suzuki, I., et al. (2005). Acanthopanax senticosus Harms as a prophylactic for MPTPinduced Parkinson's disease in rats. J. Ethnopharmacol. 97, 375-381. doi: 10.1016/j.jep.2004.11.031

Fujikawa, T., Yamaguchi, A., Morita, I., Takeda, H., and Nishibe, S. (1996). Protective effects of Acanthopanax senticosus Harms from Hokkaido and its components on gastric ulcer in restrained cold water stressed rats. Biol. Pharm. Bull. 19, 1227-1230. doi: 10.1248/bpb.19.1227

Harris, M., Farrell, V., Houtkooper, L., Going, S., and Lohman, T. (2015). Associations of polyunsaturated fatty acid intake with bone mineral density in postmenopausal women. J. Osteoporos. 2015:737521. doi: 10.1155/2015/ 737521

Hassan, N. M., Hassan, R. A., Setta, L. A., El-moniem, M. A., Ahmed, H. H., and Hammouda, F. M. (2010). Potent role of dietary phytoestrogen plants cultivated in Egypt against osteoporosis in ovariectomized rats. Aust. J. Basic Appl. Sci. 4, 359-369.

Horne, A. M., Mihov, B., and Reid, I. R. (2018). Bone loss after romosozumab/denosumab: effects of bisphosphonates. Calcif Tissue Int. 103, 55-61. doi: 10.1007/s00223-018-0404-6
Engineering Project of China Association of Traditional Chinese Medicine (QNRC2-B06), Outstanding Talents Foundation of Heilongjiang University of Chinese Medicine (2018jc01), Natural Science Foundation of Heilongiiang Province (YQ2019H030, H2016056).

\section{SUPPLEMENTARY MATERIAL}

The Supplementary Material for this article can be found online at: https://www.frontiersin.org/articles/10.3389/fphar. 2019.00553/full\#supplementary-material

Huang, L. Z., Huang, B. K., Liang, J., Zheng, C. J., Han, T., Zhang, Q. Y., et al. (2011). Antifatigue activity of the liposoluble fraction from Acanthopanax senticosus. Phytother. Res. 25, 940-943. doi: 10.1002/ptr.3346

Hui, J. Y., Choi, J. W., Mount, D. B., Zhu, Y., Zhang, Y., and Choi, H. K. (2012). The independent association between parathyroid hormone levels and hyperuricemia: a national population study. Arthritis Res. Ther. 14, R56-R56. doi: 10.1186/ar3769

Johnson, R. J., Kang, D. H., Feig, D., Kivlighn, S., Kanellis, J., Watanabe, S., et al. (2003). Is there a pathogenetic role for uric acid in hypertension and cardiovascular and renal disease? Hypertension 41, 1183-1190. doi: 10.1161/01.HYP.0000069700.62727.C5

Leb, G., Warnkross, H., and Obermayer-Pietsch, B. (1994). Thyroid hormone excess and osteoporosis. Acta Med. Austriaca. 21, 65-67.

Lee, S., Son, D., Ryu, J., Lee, Y. S., Jung, S. H., Kang, J., et al. (2004). Anti-oxidant activities of Acanthopanax senticosus stems and their lignan components. Arch. Pharm. Res. 27, 106-110. doi: 10.1007/BF02980055

Liu, C., Alessandro, A., and Xia, Y. (2017). Metabolomic approach in probing drug candidates. Curr. Top. Med. Chem.. 17, 1741-1749. doi: $10.2174 / 1568026617666161116144146$

Liu, S. P., An, J. T., Wang, R., and Li, Q. (2012). Simultaneous quantification of five bioactive components of Acanthopanax senticosus and its extract by ultra performance liquid chromatography with electrospray ionization time-of-flight mass spectrometry. Molecules. 17, 7903-7913. doi: 10.3390/molecules 17077903

Liu, X., Zhang, A., Fang, H., Li, M., Song, Q., Su, J., et al. (2018). Serum metabolomics strategy for understanding the therapeutic effects of YinChen-Hao-Tang against Yanghuang syndrome. RSC Adv. 8, 7403-7413. doi: 10.1039/C7RA11048K

Orchard, T. S., Ing, S. W., Lu, B., Belury, M. A., Johnson, K., Wactawski-Wende, J., et al. (2013). The association of red blood cell n-3 and n-6 fatty acids to dietary fatty acid intake, bone mineral density and hip fracture risk in The Women's Health Initiative. J. Bone Mineral Res. 28, 505-515. doi: 10.1002/jbmr.1772

Ren, J. L., Zhang, A. H., Kong, L., and Wang, X -J. (2018). Advances in mass spectrometry-based metabolomics for investigation of metabolites. RSC Adv. 8, 22335-22350. doi: 10.1039/C8RA01574K

Sautin, Y. Y., and Johnson, R. J. (2008). Uric acid: the oxidantantioxidant paradox. Nucleosides Nucleotides Nucleic Acids 27, 608-619. doi: 10.1080/15257770802138558

Song, Q., Zhang, A.-H., Yan, G.-L., Liub, L., and Wang, Z.-J. (2017). Technological advances in current metabolomics and its application in tradition Chinese medicine. RSC Adv. 7, 53516-53524. doi: 10.1039/C7RA02056B

Sun, H., Liu, J., Zhang, A., Zhang, Y., Meng, X., Han, Y., et al. (2016) Characterization of the multiple components of Acanthopanax Senticosus stem by ultra high performance liquid chromatography with quadrupole time-of-flight tandem mass spectrometry. J. Sep. Sci. 39, 496-502. doi: $10.1002 /$ jssc. 201500915

Sun, H., Wang, M., Zhang, A., Ni, B., Dong, H., and Wang, X. (2013). UPLC-Q-TOF-HDMS analysis of constituents in the root of two kinds of aconitum using a metabolomics approach. Phytochem. Anal. 24, 263-276. doi: $10.1002 /$ pca. 2407

Sun, H., Zhang, A., and Wang, X. (2012). Potential role of metabolomic approaches for Chinese medicine syndromes and herbal medicine. Phytother. Res. Ptr. 26, 1466-1471. doi: 10.1002/ptr.4613

Sun, H., Zhang, A. H., Liu, S. B., Qiu, S., Li, X. N., Zhang, T. L., et al. (2018). Cell metabolomics identify regulatory pathways and targets of magnoline 
against prostate cancer. J. Chromatogr. B Anal. Technol. Biomed. Life Sci. 1102-1103:143-151. doi: 10.1016/j.jchromb.2018.10.017

Sun, Y. N., Li, W., Yan, X -T., Yang, S. Y., and Kim, Y. H. (2014). Chemical constituents from the stems of Acanthopanax divaricatus var. albeofructus. Biochem. Syst. Ecol. 57, 164-168. doi: 10.1016/j.bse.2014.08.008

Szabados, L., and Savouré, A. (2010). Proline: a multifunctional amino acid. Trends Plant Sci.15, 89-97. doi: 10.1016/j.tplants.2009.11.009

Tella, S. H., and Gallagher, J. C. (2014). Prevention and treatment of postmenopausal osteoporosis. J. Ster. Biochem. Mol. Biol. 142, 155-170. doi: 10.1016/j.jsbmb.2013.09.008

Torricelli, P., Fini, M., Giavaresi, G., and Giardino, R. (2003). Human osteopenic bone-derived osteoblasts: essential amino acids treatment effects. Artif. Cells Blood Substit. Immobil. Biotechnol. 31, 35-46. doi: 10.1081/BIO-1200 18002

Wang, X., Han, Y., Zhang, A., and Sun, H. (2015a). "Metabolic profiling provides a system for the understanding of Alzheimer's disease in rats post-treatment with Kaixin San," in Chinmedomics eds K. Jones, M. McLaughlin, J. Haynes and M. Rogers (Academic Press; Mica Haley), 347-362. doi: 10.1016/B978-0-12-803117-9.00022-6

Wang, X., Liu, J., Zhang, A., Sun, H., and Zhang, Y. (2017). "Systematic characterization of the absorbed components of Acanthopanax senticosus stem," in Serum Pharmacochemistry of Traditional Chinese Medicine, eds K. Jones, M. McLaughlin, E. Taylor and M. Rogers (Mica Haley), 313-336. doi: 10.1016/B978-0-12-811147-5.00023-6

Wang, X., Lv, H., Zhang, A., Sun, W., Liu, L., Wang, P., et al. (2014). Metabolite profiling and pathway analysis of acute hepatitis rats by UPLCESI MS combined with pattern recognition methods. Liver Int.. 34, 759-770. doi: 10.1111/liv.12301

Wang, X., Sun, H., Zhang, A., Wang, P., and Han, Y. (2015b). Ultra-performance liquid chromatography coupled to mass spectrometry as a sensitive and powerful technology for metabolomic studies. J. Sep. Sci. 34, 3451-3459. doi: $10.1002 /$ jssc. 201100333

Wang, X., Wang, Q., Zhang, A., Zhang, F., Zhang, H., Sun, H., et al. (2013a). Metabolomics study of intervention effects of Wen-Xin-Formula using ultra high-performance liquid chromatography/mass spectrometry coupled with pattern recognition approach. J. Pharm. Biomed. Anal. 74, 22-30. doi: 10.1016/j.jpba.2012.10.009

Wang, X., Zhang, A., and Sun, H. (2013b). Power of metabolomics in diagnosis and biomarker discovery of hepatocellular carcinoma. Hepatology 57, 2072-2077. doi: 10.1002/hep.26130

Wang, X., Zhang, A., Sun, H., Han, Y., and Yan, G. (2016). Discovery and development of innovative drug from traditional medicine by integrated chinmedomics strategies in the post-genomic era. TrAC Trends Anal. Chem. 76, 86-94. doi: 10.1016/j.trac.2015.11.010

Wang, Y., Zhou, R., Zhong, W., Hu, C., Lu, S., and Chai, Y. (2018). Association of gout with osteoporotic fractures. Int. Orthop. 42, 2041-2047. doi: 10.1007/s00264-018-4033-5

Watts, N. B. (2018). Postmenopausal osteoporosis: a clinical review. J. Womens Health 27, 1093-1096. doi: 10.1089/jwh.2017.6706

Wauquier, F., Leotoing, L., Coxam, V., Guicheux, J., and Wittrant, Y. (2009). Oxidative stress in bone remodelling and disease. Trends Mol. Med.. 15, 468-477. doi: 10.1016/j.molmed.2009.08.004

Wu, F., Sun, H., Wei, W., Han, Y., Wang, P., Dong, T., et al. (2011). Rapid and global detection and characterization of the constituents in ShengMai San by ultra-performance liquid chromatography-high-definition mass spectrometry. J. Sep. Sci. 34, 3194-3199. doi: 10.1002/jssc.201100253

Wu, X., Zhao, C., Zhang, A., Zhang, J., Wang, X., Sun, X., et al. (2018). Highthroughput metabolomics used to identify potential therapeutic targets of Guizhi Fuling Wan against endometriosis of cold coagulation and blood stasis. RSC Adv. 8, 19238-19250. doi: 10.1039/C8RA00978C

Yan, Y., Zhang, A., Dong, H., Yan, G., Sun, H., Wu, X., et al. (2017). Toxicity and detoxification effects of herbalcaowuvia ultra performance liquid chromatography/mass spectrometry metabolomics analyzed using pattern recognition method. Pharmacogn. Mag. 13, 683-692. doi: 10.4103/pm.pm_475_16

Zhang, A., Sun, H., Qiu, S., and Wang, X. (2013a). Advancing drug discovery and development from active constituents of yinchenhao tang, a famous traditional chinese medicine formula. Evid. Based Complement. Alternat. Med. 2013:257909. doi: 10.1155/2013/257909

Zhang, A., Sun, H., Sun, W., and Wang, X. (2015b). "Metabolomics and Proteomics Annotate Therapeutic Mechanisms of Geniposide," in Chinmedomics eds K. Jones, M. McLaughlin, J. Haynes and M. Rogers (Academic Press; Mica Haley), 157-173. doi: 10.1016/B978-0-12-803117-9.00010-X

Zhang, A., Sun, H., Wang, P., Han, Y., and Wang, X. (2011). Modern analytical techniques in metabolomics analysis. Analyst 137, 293-300. doi: 10.1039/C1AN15605E

Zhang, A., Sun, H., and Wang, X. (2014c). Urinary metabolic profiling of rat models revealed protective function of scoparone against alcohol induced hepatotoxicity. Sci. Rep. 4:6768. doi: 10.1038/srep06768

Zhang, A., Sun, H., and Wang, X. (2018b). Mass spectrometry-driven drug discovery for development of herbal medicine. Mass Spectrom. Rev. 37, 307-320. doi: 10.1002/mas.21529

Zhang, A., Sun, H., Xu, H., Qiu, S., and Wang, X. (2013b). Cell metabolomics. OMICS. 17, 495-501. doi: 10.1089/omi.2012.0090

Zhang, A., Sun, H., Yan, G., Han, Y., and Wang, X. (2014b). Metabolomics analysis of marker metabolites for patients with pancreatic cancer. Med. Chem. 4, 506-510. doi: 10.4172/2161-0444.1000186

Zhang, A., Sun, H., Yan, G., Han, Y., Zhao, Q., and Wang, X.-J. (2018a). Chinmedomics: a powerful approach integrating metabolomics with serum pharmacochemistry to evaluate the efficacy of traditional Chinese medicine. Engineering 5, 60-68. doi: 10.1016/j.eng.2018.11.008

Zhang, A., Sun, H., Yan, G., Wang, P., Han, Y., and Wang, X. (2014a). Metabolomics in diagnosis and biomarker discovery of colorectal cancer. Cancer Lett. 345, 17-20. doi: 10.1016/j.canlet.2013.11.011

Zhang, A., Sun, H., Yan, G., Wang, P., and Wang, X. (2015a). Metabolomics for biomarker discovery: moving to the clinic. Biomed Res. Int. 2015:354671 doi: 10.1155/2015/354671

Zhang, A., Wang, H., Sun, H., Zhang, Y., An, N., Yan, G., et al. (2015c). Metabolomics strategy reveals therapeutical assessment of limonin on nonbacterial prostatitis. Food Function 6, 3540-3549. doi: 10.1039/C5FO00489F

Zhang, A. H., Sun, H., Yan, G. L., Yuan, Y., Han, Y., and Wang, X. J. (2014d). Metabolomics study of type 2 diabetes using ultra-performance LCESI/quadrupole-TOF high-definition MS coupled with pattern recognition methods. J. Physiol. Biochem. 70, 117-128. doi: 10.1007/s13105-013-0286-Z

Zhang, X. L., Ren, F., Huang, W., Ding, R. T., Zhou, Q. S., and Liu, X. W. (2010a). Anti-fatigue activity of extracts of stem bark from Acanthopanax senticosus. Molecules. 16, 28-37. doi: 10.3390/molecules 16010028

Zhang, Y., Zhang, A., Zhang, Y., Sun, H., Meng, X., Yan, G., et al. (2016). Application of ultra-performance liquid chromatography with time-of-flight mass spectrometry for the rapid analysis of constituents and metabolites from the extracts of Acanthopanax senticosus harms leaf. Pharmacogn. Mag. 12, 145-152. doi: 10.4103/0973-1296.177902

Zhang, Y. X., Wu, Z. H., Gao, H. Y., Huang, J., Sun, B. H., and Wu, L. J. (2010b). Isolation and identification of chemical constituents from stems and leaves of Acanthopanax senticosus (Rupr.et Maxim.) Harms. J. Shenyang Pharmac. Univ. 27, 110-112. doi: 10.14066/j.cnki.cn21-1349/r.2010.02.007

Zhao, Q., Zhang, A., Zong, W., An, N., Zhang, H., Luan, Y., et al. (2016). Chemometrics strategy coupled with high resolution mass spectrometry for analyzing and interpreting comprehensive metabolomic characterization of hyperlipemia. RSC Adv. 6, 112534-112543. doi: 10.1039/C6RA24267G

Conflict of Interest Statement: The authors declare that the research was conducted in the absence of any commercial or financial relationships that could be construed as a potential conflict of interest.

Copyright (c) 2019 Zhang, Ma, Sun, Zhang, Liu, Wu and Wang. This is an openaccess article distributed under the terms of the Creative Commons Attribution License (CC BY). The use, distribution or reproduction in other forums is permitted, provided the original author(s) and the copyright owner(s) are credited and that the original publication in this journal is cited, in accordance with accepted academic practice. No use, distribution or reproduction is permitted which does not comply with these terms. 